\title{
Selbstdispensation
}

\section{Zurückhaltende Ärzte, zufriedene Patienten}

Seit dem 1. Mai 2012 dürfen Ärzte in Zürich und Winterthur eine Praxisapotheke führen; rund 30 Prozent von ihnen beantragten bei der kantonalen Gesundheitsdirektion eine Bewilligung. Wichtigstes Motiv dabei: den Patienten eine zusätzliche Dienstleistung anbieten - was diese sehr schätzen, wie eine Umfrage von Zur Rose Suisse AG und der Ärzteorganisation Hawadoc zeigt.

Gaudenz Weber

Zur Rose Suisse AG

Korrespondenz: Gaudenz Weber Zur Rose Suisse AG Walzmühlestrasse 60 CH-8500 Frauenfeld Tel. 0527240020 gaudenz.weber[at]zurrose.ch
Fast 15 Jahre dauerte das Hin und Her um die direkte Medikamentenabgabe in Zürich und Winterthur. Nach einem letzten Entscheid des Bundesgerichts ist die Selbstdispensation im Kanton Zürich seit dem 1. Mai 2012 flächendeckend zulässig.

Ein Jahr danach wollte Zur Rose Schweiz wissen, welche Erfahrungen beim Einführen und Betreiben der Praxisapotheke gemacht wurden. Dazu befragte der Ärztegrossist zusammen mit Hawadoc die frei praktizierenden Ärztinnen und Ärzte in Zürich und Winterthur. Von den Antwortenden ( $\mathrm{n}=195)$ geben
Was diese offenbar schätzen: Alle antwortenden Ärzte erfuhren nur positive Reaktionen auf die Möglichkeit, die Medikamente direkt nach der Konsultation abgeben zu können. Dadurch steige das Vertrauen der Patienten wie auch das Verständnis für die einzunehmenden Arzneimittel. Gleichzeitig zeigen die Ärzte viel Nachsicht, wenn die Patienten weiterhin ein Rezept möchten. Stellvertretend dafür schreibt ein Arzt: «Viele meiner Patienten haben eine gute Beziehung zu ihrem Apotheker. Daran soll sich nichts ändern.»

\section{«Ärztinnen und Ärzte steigen schrittweise in die Selbstdispensation ein.»}

61 Prozent Medikamente ab, weitere 6 Prozent planen die Abgabe in nächster Zeit. Jeder Zehnte hat sich noch nicht entschieden; der Rest - rund ein Viertel - verzichtet auf die Selbstdispensation, hauptsächlich, weil der Aufwand als zu gross erachtet wird.

Wichtigstes Motiv für das Einrichten einer Praxisapotheke war, den Patientinnen und Patienten eine zusätzliche Dienstleistung zu bieten (Abb. 1).
Dazu passt auch, dass 56 Prozent der Ärzte, die eine Praxisapotheke einführten, mit wenigen Medikamenten starteten und das Sortiment erst nach und nach ausbauten. Weitere 11 Prozent halten das Lager bewusst klein und schreiben hauptsächlich Rezepte. Nur jeder Neunte hatte von Beginn weg ein umfassendes Sortiment.

Das schrittweise Vorgehen der Mehrheit verdeutlicht den Respekt, den man vor der neuen Auf-

Abbildung 1

Nennen Sie bitte die Gründe, weshalb Sie sich für die Einführung der Selbstdispensation entschieden haben. $(n=118)$

Bessere Kontrolle über die Medikation

Tiefere Gesundheitskosten dank SD

Ertrag von Medikamentenverkauf

Zusätzliche Dienstleistung für meine Patienten

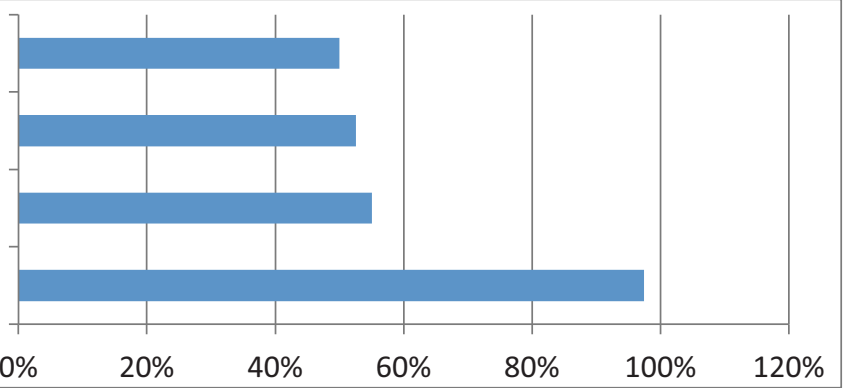


Abbildung 2

Was waren die grössten Herausforderungen bei der Einführung der Praxisapotheke? $(n=118)$

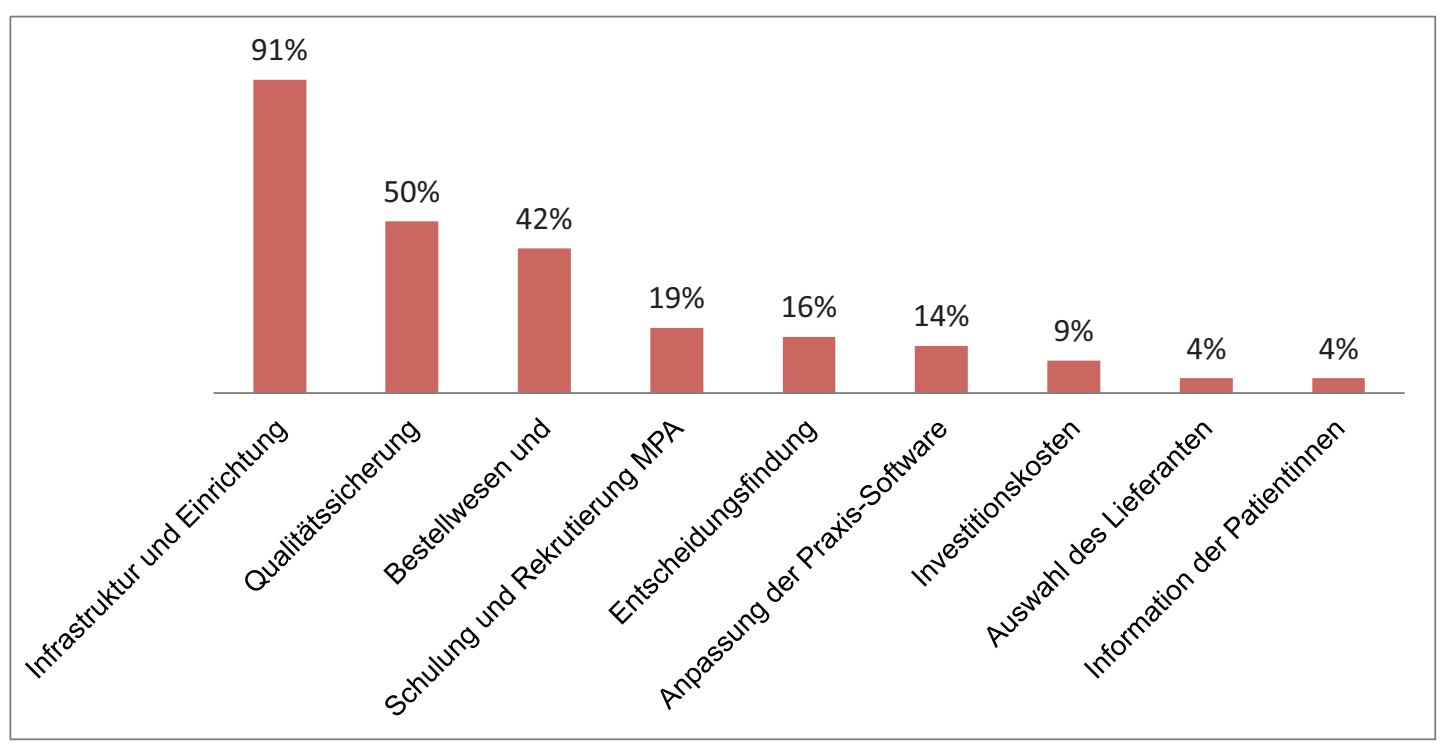

gabe hatte. Nach den grössten Herausforderungen beim Einführen der Praxisapotheke gefragt, nannten 91 Prozent die Infrastruktur und Einrichtung (Abb. 2). Besonders die Wahl des richtigen Medikamentenkühlschranks sowie die Frage, ob eine Klimaanlage nötig sei, forderten die Ärztinnen und Ärzte. Daneben verlangten die Qualitätssicherung (50 Prozent) sowie das Bestellwesen und Lagermanagement (42 Prozent) viel Aufmerksamkeit. Hier galt es vor allem, die Richtlinien der Kantonalen Heilmittelkontrolle zu erfüllen. Kaum als Hindernisse wurden die Investitionen (9 Prozent) und die Anpassung der Praxis-Software (14 Prozent) empfunden. und bei der Praxis-Software 82 Prozent. Selbst die Qualitätssicherung, von vielen als grosse Herausforderung empfunden, läuft in vier von fünf Praxen reibungslos. Dies belegen auch die Ergebnisse der ersten Prüfungen durch die Kantonale Heilmittelkontrolle: In 97 Prozent der geprüften Praxisapotheken stellte sie keine oder nur minime Mängel fest.

Aus diesen Resultaten lassen sich folgende Erkenntnisse ableiten:

Ärztinnen und Ärzte ...

- sehen die Selbstdispensation primär als Zusatzleistungen für die Patienten und nur bedingt als Mittel für mehr Einnahmen.

\section{«Ärztinnen und Ärzte sehen die Selbstdispensation primär als Zusatzleistungen für die Patienten.»}

Eine wichtige Rolle beim Einrichten einer Praxisapotheke spielen die Grossisten. 81 Prozent der Ärzte waren mit der Unterstützung durch ihre Lieferanten sehr zufrieden, weitere 11 Prozent zufrieden. Ähnlich hoch sind die Werte beim Sortiment und den Konditionen, beim Bestellwesen und der Auslieferung sowie beim Aussen- und Kundendienst.

Die Erfahrungen mit der Praxisapotheke sind nach einem Jahr durchwegs positiv; das behutsame Vorgehen bewährt sich offenkundig. Die Abrechnung der Medikamentenabgabe erfolgt für 91 Prozent der Antwortenden reibungslos; bei der Bewirtschaftung durch das MPA-Team sind es 86 Prozent
- wenden die Selbstdispensation sehr differenziert an; die Selbstbestimmung der Patienten bleibt gewahrt: Die Patientin bzw. der Patient entscheidet, ob nach der Konsultation ein Rezept oder das Medikament gewünscht ist.

- steigen schrittweise in die Selbstdispensation ein; die Qualitätssicherung hat hohes Gewicht.

- nutzen und schätzen die Unterstützung durch ihre Lieferanten.

$\mathrm{Zu}$ guter Letzt: Ärztinnen und Ärzte, die auf die Selbstdispensation verzichten, scheinen keine Nachteile zu haben. 